\title{
Nonlinear discrete light propagation in photonic liquid crystal fibers
}

\author{
Katarzyna A. Rutkowska, Urszula A. Laudyn, Paweł S. Jung \\ Faculty of Physics, Warsaw University of Technology, Koszykowa 75, PL-00662 Warsaw, Poland
}

Received March 26, 2013; accepted March 28, 2013; published March 31, 2013

\begin{abstract}
In this report, the results of numerical simulations describing (discrete) light propagation in photonic liquid crystal fibers (PLCFs) are presented. Specifically, the deviation in the spatial broadening of the beams characterized by different initial widths is analyzed. In this context, particular attention is devoted to the cases in which thermal (optical) nonlinearity in liquid crystals (LCs) is taken into account. Spatial light localization and/or delocalization are demonstrated, with the final scenario determined by the initial width and amplitude of the light beam. In particular, possibility of the discrete soliton generation is demonstrated. A qualitative comparison with experimental data obtained in analogous conditions (with use of specific PLCF samples) is also made.
\end{abstract}

Photonic crystal fibers (PCFs), i.e. structures composed of $2 \mathrm{D}$ photonic crystals elongated homogeneously over the propagation direction [1], have hitherto found a wide range of potential applications in many areas of science and technology [2]. Thanks to their unique features, when compared to conventional optical fibers, PCFs are successfully used for optical communication and sensing. In recent years, PCFs have emerged also as active fibers (i.e. for fiber lasers and fiber amplifiers). Solid-core PCFs (of specific design) are widely used (also commercially) for the supercontinuum generation.

The cross-sections of PCFs are typically formed by the periodic wavelength-scale matrix (square or triangular) of microscopic air-holes located in the solid material (e.g. silica, high-index/multicomponent glasses, silver-halide crystalline materials, and polymers) [2]. One of the most important advantages offered by PCFs is the high flexibility of their design. Specifically, by changing: (i) the geometry of a 2D periodic structure (described by the air-holes shape, size and arrangement) and (ii) the material properties (e.g. component material(s), size and material(s) out of which the core is made), it is possible to design and control the guiding properties of PCFs. In this way, two different mechanisms for the light guiding - i.e. the photonic bandgap (PBG) guiding and the index guiding (here based on the modified total internal reflection, mTIR) can be respectively observed in PCF structures of specific geometry. It has been also shown that the guiding properties of PCF can be additionally adjusted (also dynamically) by introducing fluid and/or gaseous materials into the air-holes. Specifically, the application of liquid crystals (LCs) has resulted in a new type of photonic structures, typically referred to as

\footnotetext{
*E-mail: kasia@if.pw.edu.pl
}

photonic liquid crystal fibers (PLCFs). High tunability of PLCFs (manifested e.g. by tuning of PBGs and switching between the guiding mechanisms) [3] arises from unique optical properties of LCs [4]. The latter can be dynamically controlled by external fields and factors and, what is particularly important in the context of this letter, by the optical beam itself (when nonlinear phenomena in LCs are considered).
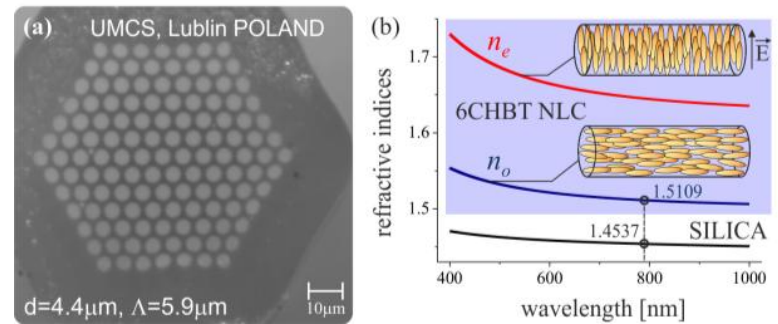

Fig. 1. (a) Microscope image of the host PCF (fabricated at Marie Curie-Sklodowska University in Lublin), where $d$ is the diameter of the air-hole and $\Lambda$ is the pitch. (b) Refractive indices of 6CHBT NLC (at $20^{\circ} \mathrm{C}$ ) as a function of the wavelength [5], where $n_{o}$ is the ordinary and $n_{e}$ is the extraordinary refractive index. The refractive index of silica (at the same temperature) is also shown for comparison.

In the PCF applied in this work (see Fig. 1a for the cross-section), a uniform hexagonal lattice of the air-holes is distributed in the silica cladding. This regularity of a periodic structure [i.e. the lack of $\operatorname{defect}(\mathrm{s})$ in the lattice] is the exact opposite of typical solid-core PCFs. This particular PCF geometry [to be infiltrated with 6CHBT nematic liquid crystal (NLC) whose refractive indices are higher than those of the silica glass; see Fig. $1 \mathrm{~b}$ for the chromatic dispersion [5])] can be considered as a matrix of waveguide channels and has been chosen to study discrete light propagation in analogical conditions as it has been demonstrated in uniform waveguide matrices. So far, the two-dimensional lattices of evanescently coupled waveguides have been demonstrated in photorefractive materials, and as the cubic and hexagonal waveguide arrays written (with fs-laser pulses) in fused silica [6-8]. In a linear regime, the wavelength-dependent coupling between adjacent waveguide channels has been presented, while nonlinear dynamics has been also developed, giving thus rise to all-optical switching and routing (based on two-dimensional solitons) [6-8 and Ref. therein]. It can be shown that in such photonic structures, the output intensity profile strongly depends on optical and geometrical parameters of the system (including 
wavelength, beam-size, lattice arrangement and index contrast) [6-9]. The advantage in application of a PLCFbased waveguide matrix stems from the possibility of dynamical changes in the light propagation (e.g. by applied fields and factors). Specifically, the introduction of nonlinear effects (related to reorientational and thermal nonlinearity of NLCs) may result in dramatic changes in propagation characteristics (e.g. when discrete soliton is observed).

In the PLCF samples tested in our experiment, a special procedure based on photoalignment (with use of the SE130 polymer) [10] allowed to introduce planar anchoring on the capillaries walls. It is why we decided to take the value of the ordinary refractive index of NLC in our calculations.

Numerical algorithms applied in this letter are based on the fundamental paraxial propagation equation for electric field amplitude E:

$-2 i \beta \frac{\partial}{\partial z} \mathrm{E}+\frac{\partial^{2}}{\partial x^{2}} \mathrm{E}+\frac{\partial^{2}}{\partial y^{2}} \mathrm{E}+\gamma^{2} \mathrm{E}=0$,

with $\gamma$ accounting for the (linear and nonlinear) spatial refractive index distribution:

$\gamma^{2}=\frac{\omega^{2}}{c^{2}}\left(n^{2}+2 \alpha n|E|^{2}\right)-\beta^{2}=\frac{\omega^{2} n^{2}}{c^{2}}\left(1+\frac{n_{2} I}{Z}\right)-\beta^{2}$,

where: $Z$ - impedance of free space, $n=n(x, y)$ - linear refractive index, I - light intensity, $\alpha$ - nonlinear and $n_{2}$ Kerr coefficient, respectively. For NLCs both a positive $\left(\mathrm{n}_{2}>0\right)$ and negative $\left(\mathrm{n}_{2}<0\right)$ Kerr-type nonlinearity occurs due to the molecular reorientation and thermal effects, respectively [4]. In this letter we limited our analysis to the case of negative Kerr-type nonlinearity (as dedicated by preliminary experimental observations).

In our numerical simulations a homogenous distribution of the refractive index inside LC rods (taken as $n_{\mathrm{o}}$ ) was assumed. A finite difference scheme (with a square mesh grid of $0.1 \mu \mathrm{m})$ was applied. The calculation window was set to $100 \mu \mathrm{m} \times 100 \mu \mathrm{m}$ to enclose the whole PLCF structure (note that only the central part of the calculation window is shown in Figs. 2-5).

constant (low, i.e. $\alpha \equiv 0$ ) optical power; $\mathrm{z}$ increases

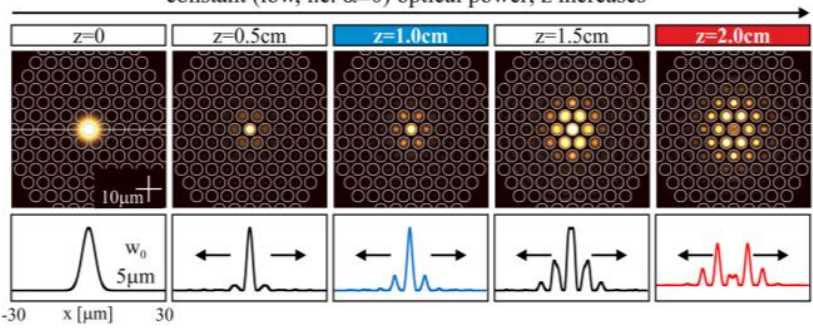

Fig. 2. Numerical results for a low-power Gaussian beam with an initial width of $5 \mu \mathrm{m}$ and the wavelength of $793 \mathrm{~nm}$, showing discrete diffraction of the beam launched into the center of the waveguide matrix. In the lower row, the (normalized intensity) beam transverse profiles at the specific propagation distances are presented. The black arrows indicate how the beam is spreading in space on the way of propagation.
Figure 2 shows the light propagation in the linear regime (i.e. for $\alpha \equiv 0$ ), when a Gaussian beam with the initial waist of $5 \mu \mathrm{m}$ is launched into the center of a periodic structure. The light is coupling and spreading into an increasing number of LC waveguide channels. Analogical results obtained for beams with different initial widths are presented in Fig. 3. As one can see, more waveguide channels illuminated at the input facet, less visible are features and effects characteristic for the discrete diffraction (see e.g. last row in Fig. 3).

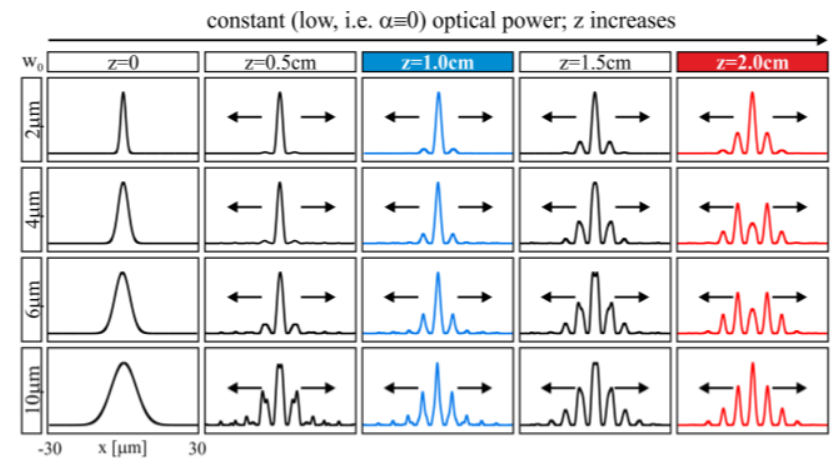

Fig. 3. Numerical results showing discrete diffraction of the Gaussian beams with the different initial widths $\mathrm{w}_{0}$.

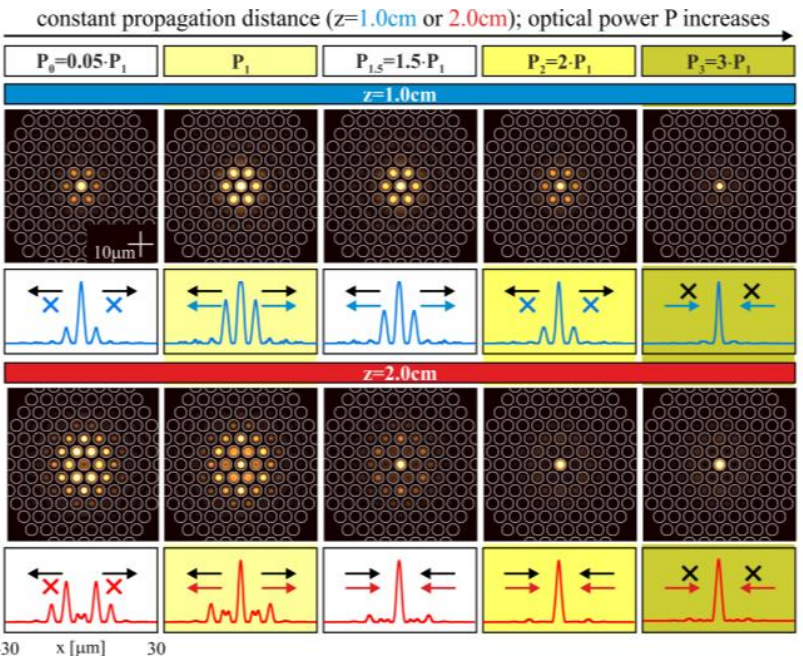

Fig. 4. Numerical results (for Gaussian beam with an initial width of $5 \mu \mathrm{m})$ showing how discrete light propagation changes with power when negative (defocusing) Kerr nonlinearity is assumed. In the current notation $\left|\mathrm{n}_{2}\right| \mathrm{P}_{1}=7.85 \cdot 10^{-4} \mu \mathrm{m}^{2}$. Black marks (arrows and crosses) shows how the beam width changes on the way of propagation (for the fixed power), while the color ones indicates (for specific value of power) how the beam width changes with respect to the linear case (for the same propagation distance).

Going from the linear to nonlinear regime, when the power of a propagating beam increases, the refractive index of the excited waveguide channels (LC cores) is modified by the nonlinearity, and discrete diffraction can be effectively counteracted by the power-induced detuning of the launch channel(s). In the case of defocusing (negative) Kerr nonlinearity (which corresponds to the thermal nonlinearity in the case of 
NLCs), initially, when optical power gets higher, the index contrast decreases and a wider broadening of the beam is observed (due to the coupling distance getting shorter). However, further power growth leads to a situation in which the propagation constant of the central LC channel significantly differs from the others. As a result, light localization is observed, and finally (if the power is high enough) the discrete soliton [7] (here for $\left|\mathrm{n}_{2} \mathrm{P}\right|>2.35 \cdot 10^{-3} \mu \mathrm{m}^{2}$ for $\left.\mathrm{w}_{0}=5 \mu \mathrm{m}\right)$ propagates in the central LCs channel. In principle, the narrower is the beam at the output, the lower optical power is required for each phase in the delocalization and localization processes described above (please compare results shown in Figs. 4-5).

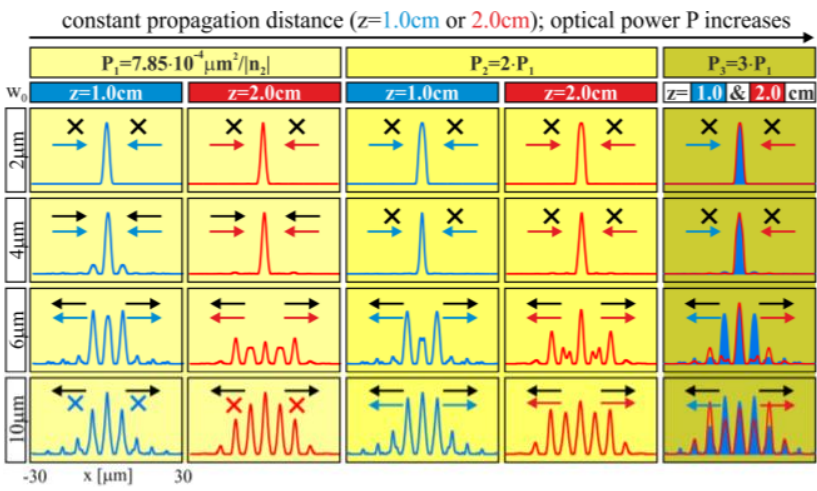

Fig. 5. Numerical results (for different initial beam widths) showing how discrete light propagation changes with power. Defocusing Kerrtype nonlinearity is assumed. Marks indicating how the beam width is changing with propagation distance and power are adopted as above.

The above numerical results can be confirmed experimentally when suitable measurements are performed. The experiments were performed in a 1- and 2-cm-long piece of PLCF. The linearly polarized light beam from a Ti:Sapphire laser $(\lambda=793 \mathrm{~nm})$ was coupled into the sample by means of the long-working-distance microscope objectives $(10,20$ or $50 x)$. The output intensity profile was imaged on CCD1 (by output objective), while the input facet of PLCF was imaged on CCD2 (thanks to the beam-splitter and the coupling objective). Owning to the limit of experimental means, direct quantitative comparison is not quite accessible. Instead, qualitative evaluation of the experimental and theoretical results may be performed. The influence of thermal nonlinearity on PLCF under test can be clearly observed in the experimental results (see Fig. 6b for details). Specifically for $\mathrm{w}_{0}=5 \mu \mathrm{m}$, it can be seen that firstly a slight spatial light delocalization is observed, later followed by light localization (manifested by high light intensity in the single LC channel) when the power constantly increases (as predicted theoretically).
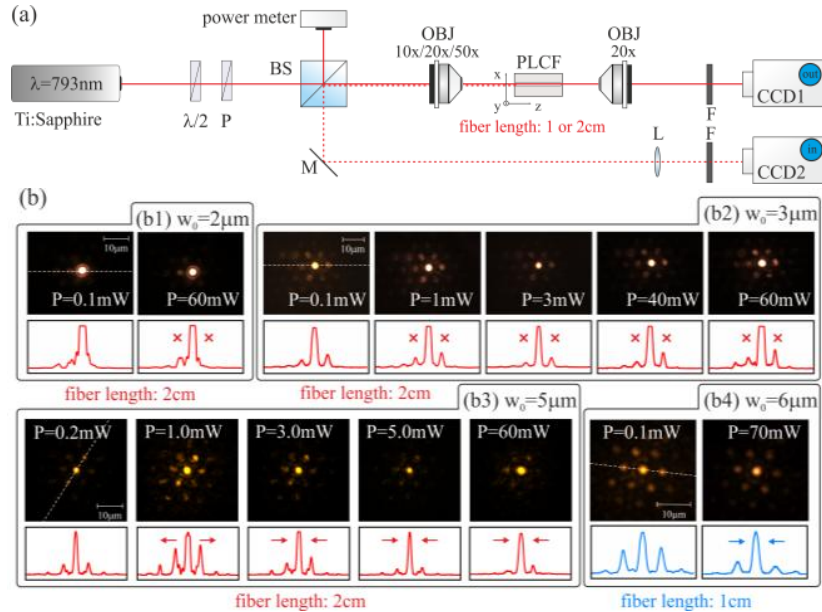

Fig. 6. (a) Experimental setup, where: $\lambda / 2$ - half-wave plate, $\mathrm{P}-$ polarizer, BS - beam-splitter, L- lens, F- neutral density filters, CCD1,2 - CCD cameras. (b) Experimental results showing the powerdependence of the output intensity profiles (at the output facet of 1- or 2-cm-long PCF infiltrated with 6CHBT NLC) for different input beam widths. Measurements were made at constant (room temperature) with no light polarization dependence noticed in experimental conditions. Please note that the scales in the images change in each photosequence and that the intensity of registered images was controlled (normalized) with the use of the neutral density filters.

In this letter we show theoretical and experimental work on the nonlinear discrete light propagation in PLCF. Experimental observations how the intensity pattern at the output facet of PLCF changes with power (for different input beams) are in a qualitative agreement with the results of numerical simulations. Specifically, taking the advantage of the thermal nonlinearity in NLCs, the powerdependent spatial light delocalization and localization (with particular form of discrete soliton) was demonstrated. Such a feature allows for all-optical switching and routing to be potentially developed in PLCFs.

The authors would like to acknowledge Homing Plus Programme (HOMING PLUS/2010-2/11) granted by the Foundation for the Polish Science and co-financed by the European Regional Development Fund. The authors would like to thank Dr. P. Mergo (for PCF samples), Prof. R. Dąbrowski (for NLC) and Dr. M. Chychłowski (for NLC orientation within PCFs).

\section{References}

[1] P. St. J. Russell, Science 299, 358 (2003).

[2] E. Poli, A.Cucinotta, S. Selleri, Photonic Crystal Fibers. Properties and Applications (Springer, The Netherlands 2007).

[3] T.R. Woliński et al., Phot. Lett. Poland 3(1), 20 (2011)

[4] I-C. Khoo, S-T. Wu, Optics and Nonlinear Optics of Liquid Crystals (World Scientific Pub., Singapore 1993).

[5] J. Schirmer et al., Mol. Cryst. Liq. Cryst. 307(1), 17 (1997).

[6] J. Fleischer, M. Segev, N. Efremidis, D. Christodoulides, Nature 422, 147 (2003)

[7] F. Lederer, Y. Silberberg, Opt. Photon. News 2, 48 (2002).

[8] A. Szameit, D. Blömer, J. Burghoff, Appl. Phys. B 82, 507 (2006).

[9] K.A. Rutkowska, K. Orzechowski, Proc. SPIE 8697, 86971F (2012)

[10] M.S. Chychłowski et al., Acta Phys. Pol. A, 118, 1100 (2010). 